\title{
Enhancing Random Forests Performance in Microarray Data Classification
}

\author{
Nicoletta Dessì, Gabriele Milia, Barbara Pes \\ Università degli Studi di Cagliari, Dipartimento di Matematica e Informatica, \\ Via Ospedale 72, 09124 Cagliari, Italy \\ \{dessi,milia.ga,pes\}@unica.it
}

\begin{abstract}
Random forests are receiving increasing attention for classification of microarray datasets. We evaluate the effects of a feature selection process on the performance of a random forest classifier as well as on the choice of two critical parameters, i.e. the forest size and the number of features chosen at each split in growing trees. Results of our experiments suggest that parameters lower than popular default values can lead to effective and more parsimonious classification models. Growing few trees on small subsets of selected features, while randomly choosing a single variable at each split, results in classification performance that compares well with state-of-art studies.
\end{abstract}

Keywords: Microarray data classification, Random Forests, Feature selection.

\section{Introduction}

As observed in [1], the random forest performance tends to decline when the number of features is huge and the proportion of truly informative features is small, such as with gene expression data. Thus, applying random forests in microarray data analysis presents an interesting research goal due to the additional issue of reducing the contribution of trees whose nodes are populated by non-informative features.

Pre-filtering features is a popular procedure that has proved to be useful to face the curse of dimensionality of gene expression data. When applied before growing a random forest, this process has to face an additional issue: asserting values for the two critical parameters of the random forest, i.e. the number of variables randomly chosen at each split, namely mtry, and the number of the trees in the forest, namely ntree.

This paper evaluates the effects of a filtering process on the predictive performance of a random forest classifier as well as on the choice of its critical parameters. Using two popular microarray datasets, we carried out classification experiments by growing random forests both on the whole set of features and on different subsets of prefiltered features: different parameter settings were explored in order to investigate the optimal trade-off between the number of trees and the number of variables randomly chosen at each split. Our results suggest that growing few trees on small subsets of pre-filtered features, with only one variable randomly chosen at each split, presents results which compare very well with state-of-art studies in literature. 


\section{Experiments and Results}

We experimented with two public microarray datasets: Leukemia [2] and Colon [3]. The overall analysis was performed using the Weka data mining environment [4]. For performance estimation, we used a standard cross-validation procedure (LOOCV), as in the majority of the papers, though it has been observed that a cross-validation setting can produce overoptimistic results on small sample size domains [5]. The performance was evaluated using the AUC (area under the ROC curve) metric in order to synthesize the information of sensitivity and specificity.

The experiments were divided into two classes:

1. Tuning on the whole dataset. We grew different random forests within the following parameters values: (i) ntree $=10,20,30,50,100,200,300,500,1000$, 1500; (ii) $m$ try $=1,2,3,5,10,20,30,40,50,80$. Both the choices (i) and (ii) aim to finely explore parameters values smaller than the common default values.

2. Tuning on filtered subsets. First, we ranked the features of the original dataset using two popular ranking methods, i.e. Information Gain (IG) and Chi Squared $\left(\chi^{2}\right)$. Based on their outputs, we selected different subsets of highly-ranked features denoted in the following as TOP10 (i.e. the first 10 top-ranked features), TOP20 (i.e. the first 20 top-ranked features) and so on. Then, we used these subsets for growing random forests within the following parameter configurations: (i) ntree $=10,20,30$, 50, 100, 200, 300; (ii) mtry = 1, 2, 3, 5, 10, 20, 30.

Results about tuning on the whole dataset. Fig.1 and Fig. 2 show, for different values of mtry, the effects of changes in the parameter ntree on the AUC. As asserted by [6], the behavior of AUC is asymptotic: as the number of trees increases, the AUC value converges to a limit. Interestingly, in both Leukemia and Colon, we observed this asymptotic trend for ntree $>100$, while previous studies [7][8] on microarray datasets made use of ntree values in the order of thousands. Globally, results in Fig. 1 and Fig. 2 suggest that, even on high-dimensional domains, the choice ntree $=100$ can be quite adequate, with further increases having negligible effects and smaller values leading to more unstable AUC performance.

As regards the influence of mtry parameter on random forest behavior, Fig. 1 and Fig. 2 show that, for small values $(\leq 50)$ of ntree, the choice of high values of mtry (mtry $>=30$ for Leukemia and mtry $>=5$ for Colon) results in higher values of AUC. This seems to suggest that, when we choose to grow a forest with a small number of trees, we need to set higher values for mtry in order to increase the probability of randomly selecting informative variables. On the other hand, if the forest is sufficiently large (ntree $>=100$ ), the influence of mtry parameter decreases. In particular, no improvement in AUC performance can be observed when setting values of mtry $>20$ and mtry $>10$ for Leukemia and Colon respectively. Hence, as previously observed for the ntree parameter, the common default setting of mtry = sqrt(M) [7][8], where $M$ is the dataset dimensionality, seems to be unnecessary large, with smaller values ensuring a good predictive performance at a lower computational cost. 

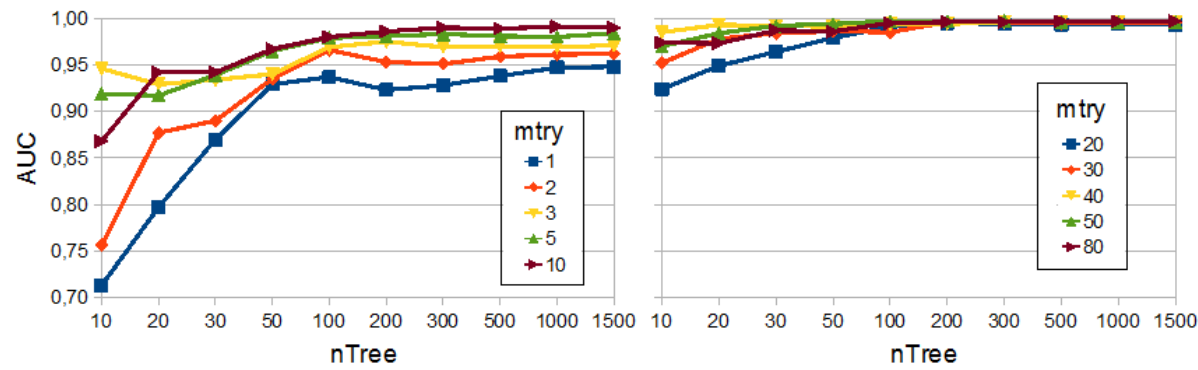

Fig. 1. Tuning on Leukemia dataset: AUC versus ntree for $m$ try $=1,2,3,5,10$ (left) and mtry $=$ $20,30,40,50,80$ (right).
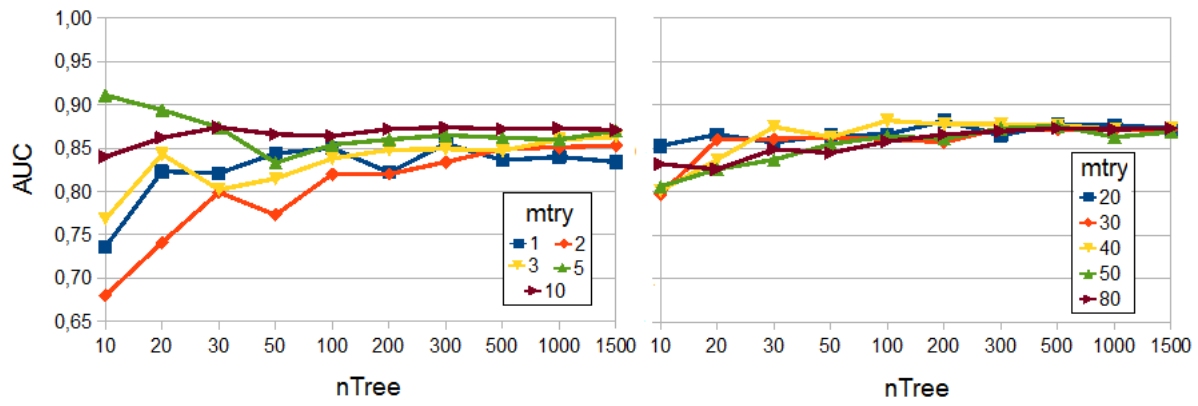

Fig. 2. Tuning on Colon dataset: AUC versus ntree for $m$ try $=1,2,3,5,10$ (left) and mtry $=20$, $30,40,50,80$ (right).

Results about tuning on filtered subsets. As said before, we applied two ranking methods (IG and $\chi^{2}$ ) and, for each ranking method, we performed tuning experiments on pre-filtered subsets of increasing size (TOP10, TOP20, etc). Table 1 summarizes the "optimal" values of both parameters $n t r e e$ and $m t r y$, i.e. the lowest values leading, on a given subset, to the best AUC result. As we can see, in most cases, the value $m t r y=1$ is sufficient to maximize the predictive performance of random forests. The optimal number of trees is also quite low, especially for Leukemia, where the AUC is maximized with at most 30 random trees. More trees (a few hundred at most) can be needed for Colon which is recognized to be a more noisy dataset. Results in Table 1 globally confirm what previously observed on the overall datasets: parameter values lower than common default values can lead to effective and more parsimonious classification models. Although surprising, the goodness of the choice mtry $=1$ is also supported (for datasets of low-moderate dimensionality, as the pre-filtered datasets here considered) by some considerations reported in [6].

Additionally, the pre-filtering process significantly improves the predictive performance. As regards Leukemia, our experiments gave excellent AUC results in all the subsets from TOP10 to TOP500. Only for larger subsets (TOP1000), the AUC decreases if the number of random trees is not sufficiently large, as we can see in Fig. 3.a, where the AUC behavior is shown for some subsets filtered by IG (an analogous trend has been registered for $\chi^{2}$ ) within the "optimal" setting $m$ try $=1$. 
Table 1. Optimal values of mtry and ntree for pre-filtered subsets of increasing size, as obtained by IG and $\chi^{2}$ ranking methods, for both Leukemia and Colon datasets.

\begin{tabular}{|c|c|c|c|c|c|c|c|c|}
\hline \multirow{2}{*}{$\begin{array}{c}\text { Pre- } \\
\text { filtered } \\
\text { subset }\end{array}$} & \multicolumn{4}{|c|}{ Leukemia } & \multicolumn{4}{c|}{ Colon } \\
\cline { 2 - 9 } & \multicolumn{2}{|c|}{ IG } & \multicolumn{2}{|c|}{$\boldsymbol{\chi}^{\mathbf{2}}$} & \multicolumn{2}{|c|}{ IG } & \multicolumn{2}{c|}{$\boldsymbol{\chi}^{\mathbf{2}}$} \\
\hline & $\boldsymbol{m}$ try & ntree & mtry & ntree & mtry & ntree & mtry & ntree \\
\hline TOP10 & 1 & 30 & 1 & 20 & 1 & 30 & 10 & 20 \\
\hline TOP20 & 1 & 10 & 1 & 10 & 1 & 10 & 1 & 200 \\
\hline TOP30 & 1 & 10 & 1 & 10 & 1 & 20 & 1 & 10 \\
\hline TOP50 & 1 & 10 & 1 & 20 & 10 & 10 & 1 & 10 \\
\hline TOP100 & 1 & 20 & 1 & 20 & 1 & 100 & 1 & 200 \\
\hline TOP300 & 1 & 30 & 1 & 20 & 1 & 100 & 1 & 300 \\
\hline TOP500 & 1 & 10 & 1 & 20 & 1 & 200 & 3 & 50 \\
\hline
\end{tabular}

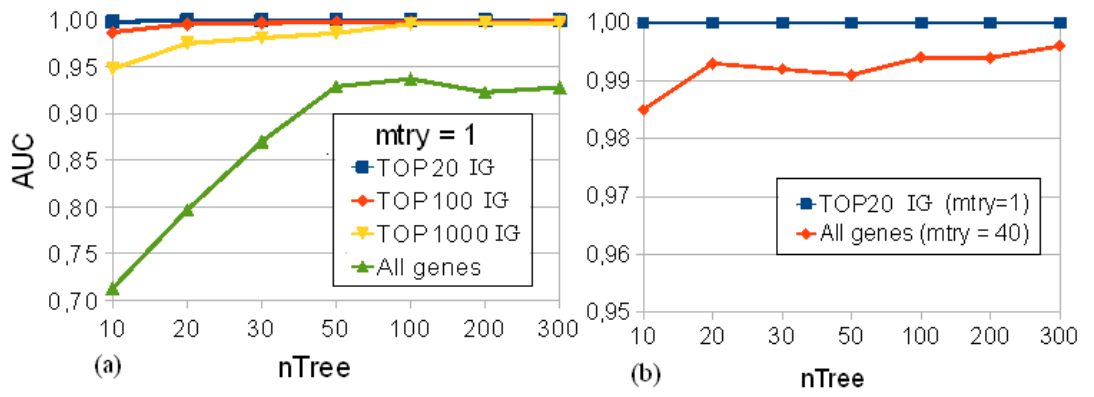

Fig. 3. Leukemia dataset: (a) AUC versus ntree for some pre-filtered subsets and for the whole dataset (mtry $=1$ for all the curves); (b) AUC versus ntree for the subset TOP20 (mtry $=1$ ) and for the whole dataset $(m t r y=40)$.

Again, we notice the asymptotic behavior of AUC. The effectiveness of prefiltering is considerable as the random forests grown on the reduced subsets greatly outperform the random forests built on the original dataset. However, the setting mtry $=1$, optimal for the filtered subsets, is not so optimal for the whole dataset, where the best AUC performance is registered for mtry $\geq 30$, as shown in Fig.1. Hence, a further demonstration of the effectiveness of the pre-filtering process is given in Fig. 3.b where the performance on the TOP20 subset (mtry = 1) is compared with the performance on the whole dataset, based on mtry $=40$ (this value corresponds to the "best" AUC curve in Fig.1). The advantages deriving from pre-filtering are confirmed by the analysis on Colon dataset (here omitted for the sake of space).

Finally, Table 2 shows the effectiveness of our approach when compared to the most cited studies that applied random forests to microarray data [7] [8]. In particular, [8] reports an error rate of 0,051 for the Leukemia dataset (in a slightly different version) using the random forest method with mtry $=\operatorname{sqrt}(\mathrm{M})$ and $n$ tree $=5000$ and without a preliminary gene selection. Within the same settings, the error rate reported for Colon is 0.127 . By integrating a variable selection approach, the best error rates given in [8] for Leukemia and Colon are 0,075 and 0,159 respectively. In [7], the AUC performance for Colon is 0.867 on the full dataset and 0,917 with gene 
selection; here, the best-performing configuration is selected among the following values of parameters: $n$ tree $=500,1000,2000$ and $m$ try $=0,5 \cdot \mathrm{sqrt}(\mathrm{M}), 1 \cdot \operatorname{sqrt}(\mathrm{M})$, $2 \cdot \operatorname{sqrt}(\mathrm{M})$.

Table 2. Our best results on Leukemia and Colon, both in terms of AUC and accuracy.

\begin{tabular}{|c|c|c|c|c|}
\hline \multirow{2}{*}{ Dataset } & \multicolumn{2}{|c|}{ On the full set of genes } & \multicolumn{2}{c|}{ Using a filtered subset } \\
\cline { 2 - 5 } & AUC & Accuracy & AUC & Accuracy \\
\hline Leukemia & 0,997 & 0,986 & 1,00 & 1,00 \\
\hline Colon & 0,911 & 0,855 & 0,939 & 0,903 \\
\hline
\end{tabular}

Acknowledgments. This research was supported by RAS, Regione Autonoma della Sardegna (Legge regionale 7 agosto 2007, n. 7), in the project "DENIS: Dataspaces Enhancing the Next Internet in Sardinia".

\section{Conclusions}

The experimental analysis performed on two public microarray datasets reveals that a pre-filtering process positively impacts both on random forest performance and on its optimal parameterization, leading to very effective and more parsimonious classification models. Our future research will address a further potentiality of the random forest method: it can be used not only for classification but also for feature selection, due to its capacity of deriving a variable importance index.

\section{References}

1. Amaratunga, D., Cabrera, J., Lee, Y.S.: Enriched random forest. Bioinformatics, vol.24, pp. 2010--2014 (2008)

2. Golub, T.R., et al.: Molecular Classification of Cancer: Class Discovery and Class Prediction by Gene Expression Monitoring. Science, 286:531--537 (1999)

3. Alon, U., et al.: Broad patterns of gene expression revealed by clustering analysis of tumor and normal colon tissues probed by oligonucleotide arrays. PNAS, vol. 96, pp. 6745--6750 (1999)

4. http://www.cs.waikato.ac.nz/ml/weka/

5. Braga-Neto, U. and Dougherty, E.: Is cross-validation valid for small-sample microarray classification? Bioinformatics, 20, pp. 374--380 (2004)

6. Breiman, L.: Random forests. Machine Learning, vol. 45, pp. 5--32 (2001)

7. Statnikov, A., Wang, L., Aliferis, C.F.: A comprehensive comparison of random forests and support vector machines for microarray-based cancer classification. BMC Bioinformatics 9:319 (2008)

8. Dìaz-Uriarte, R., Alvarez de Andrés, S.: Gene selection and classification of microarray data using random forest. BMC Bioinformatics 7:3 (2006) 